\title{
UNA VERSIÓN ESPAÑOLA DE LA KĀFIYA DE IBN AL-HĀĞIB (570/1174-5 - 646/1249) CONSERVADA EN LA BIBLIOTECA NACIONAL DE ESPAÑA
}

\section{AN UNPUBLISHED METHOD FOR LEARNING ALGERIAN ARABIC WRITTEN IN MOSTAGANEM IN THE 1970S: PRESENTATION, CONTEXT AND LINGUISTIC STUDY}

\author{
نسخة إسبانية لمخطوطة الكافية لابن الحاجب (570هـ/1147م) ححفوظة بالمكتبة الوطنية بإسبانيا
}

\author{
Maravillas Aguiar Aguilar* \\ Universidad de La Laguna
}

BIBLID [1133-8571] 28 (2021) 105.1-14

Recibido: 22/04/21 Aceptado: 09/10/21

Resumen: La Biblioteca Nacional de España conserva el ejemplar de la edición impresa de Roma de 1592 de la

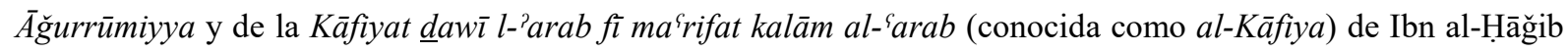
que perteneció a la biblioteca de los condes de Miranda y a Pedro de Castro (m. 1623), arzobispo de Granada y Sevilla. Este libro, catalogado como manuscrito árabe $\mathrm{n}^{\circ} 7887$, contiene varias hojas de papel escritas a mano, anotaciones manuscritas en los márgenes y escritura interlineal, presentando todo ello diversos estados de traducción al español de ambas gramáticas. Este artículo se dedica a contextualizar este ejemplar. Se concluye que tanto este libro, como otros documentos que pertenecieron al conde de Miranda, revelan dimensiones más amplias que una mera presencia de unas gramáticas de la lengua árabe en su biblioteca, dimensiones que tienen que ver con la formación del Orientalismo europeo en su especificidad española.

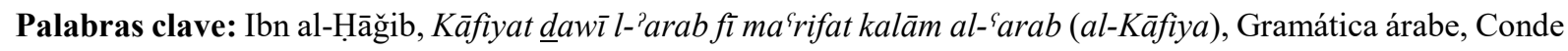
de Miranda, Pedro de Castro, Orientalismo español, Orientalismo europeo.

\begin{abstract}
The National Library of Spain preserves a sample of the Rome 1592 printed edition of the Āgurrümiyya and the Kāfiyat dawì l-'arab fi ma'rifat kalām al-'`arab (known as al-Käfiya) by Ibn al-Hāğgib that belonged to the library of the counts of Miranda and to Pedro de Castro (d. 1623), archbishop of Granada and Seville. This book, cataloged as Arabic Manuscript No. 7887, contains several sheets of paper, manuscript annotations in the margin and interlinear writing, all presenting different states of translation of both grammars. This paper focuses on the contextualization of this piece. We conclude that both this book and other documents that belonged to the Count of Miranda reveal broader dimensions than a mere presence of some grammars of the Arabic language in his library, in connection with the construction of the European Orientalism in its Spanish specificity.
\end{abstract}

Key words: Ibn al-Ḥāğib, Kāfiyat dawì l-’arab fì ma'rifat kalām al-`arab (al-Kāfiya), Arabic grammar, Count of Miranda, Pedro de Castro, Spanish Orientalism, European Orientalism.

ملخص: في المكتبة الوطنية الإسبانية توجد نسخة من نص الآجرومية لابن اجروم (الذي حقق وصدر بروما عام 1592) وكافية ذوي الأرب المباب

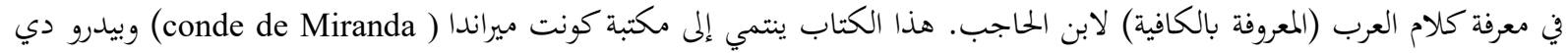

* Email: maguiar@ull.edu.es. ORCID https://orcid.org/0000-0001-8500-5465 


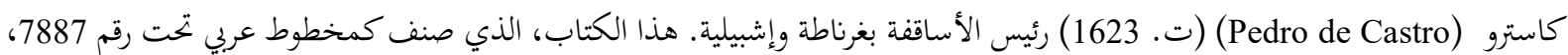

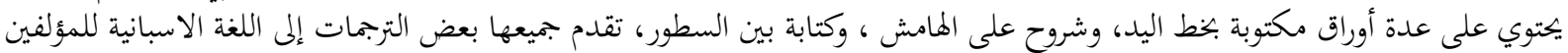

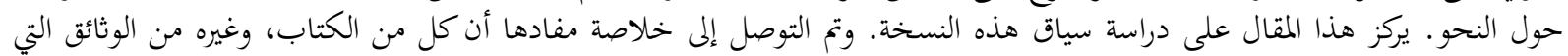

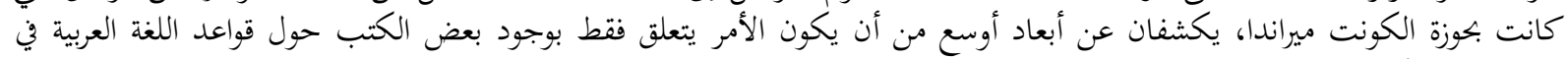

$$
\begin{aligned}
& \text { مكتبته، وهى أبعاد تتيح لنا معرفة المزيد عن طبيعة تكورين عن أبعاد أوسع من أنشراق الأوروبي الأبر في نسخته الاسبانية. } \\
& \text { كلمات مفاتيح: ابن الحاجب. كافية ذوي الأرب في معرفة كلام العرب (المعروفة بالكافية). النحو العربي. كونت ميراندا. بيدرو دي كاسترو. } \\
& \text { الاستشراق الإسباني. الاستشراق الأوروبي. }
\end{aligned}
$$

\section{Introducción}

Las gramáticas de Ibn al-Hāğğib ${ }^{(1)}$ y de Ibn Āğurrūm ${ }^{(2)}$, escritas entre los siglos VII/XIII y VIII/XIV, son gramáticas normativas de enfoque funcional que se elaboraron con la finalidad de servir como métodos de enseñanza de la lengua árabe clásica considerada correcta (fașịh) y exenta de error lingüístico (laḥn). Abū ${ }^{\varsigma} A m r{ }^{\complement} U \underline{t}$ ān b. ${ }^{\complement} U m a r$ al-Kurdī (Ibn al-Hāăğib), alfaquí mālikī y teólogo, es conocido por ser el autor de dos obras gramaticales de gran difusión: alŠăfiya y al-Kăfiya ${ }^{(3)}$. Su Kāfiya fue una de las gramáticas de la lengua árabe más utilizadas para la enseñanza de esta lengua en el islam oriental, a juzgar por los numerosos comentarios que se localizan en especial en la región turco-iraní y en Asia central, siendo muy popular en la India. Afirma Sartori (2013: 68) que su uso no parece haber sido relevante en el Magreb. Sin embargo, se constata que la gramática de Ibn Āğurrūm, conocida como Āğurrūmiyya, tuvo una amplia difusión en el islam occidental, pero no en el islam oriental ${ }^{(4)}$.

Por otro lado, se trata de dos gramáticas de la lengua árabe que tienen relación con la enseñanza del árabe en Europa a partir del siglo XVI (Aguiar Aguilar 2019). Así, veremos que el catalogado por Guillén Robles como manuscrito árabe $n^{0} 7887$ de la Biblioteca Nacional de España $^{(5)}$ es en realidad un ejemplar anotado de la edición de Roma de 1592 de la gramática de Ibn Āğurrūm, o Ăğurrūmiyya ${ }^{(6)}$, y de la Kăfiya de Ibn al-Hāăğib ${ }^{(7)}$, impresas juntas y por este orden, que contiene texto manuscrito (en hojas sueltas ${ }^{(8)}$, interlineal ${ }^{(9)}$ y al margen) ${ }^{(10)}$. Sartori y Versteegh han indicado que ambas gramáticas conocieron destinos diferentes durante siglos en el espacio lingüístico árabe, observándose una distribución geográfica que las sitúa como métodos de enseñanza de la lengua árabe en localizaciones bastante bien definidas.

(1) Nacido en Esna (Isnā) (Alto Egipto) después de 570/1174-5 y fallecido en Alejandría en 646/1249. GAL (I, 367-373), SI (531-539) y Fleisch (1986).

(2) Nacido en Fez en 672/1273-4 y fallecido en Fez en 723/1323. Ben Cheneb (1913-1936) y Troupeau (1986).

(3) Se conserva un gran número de copias de la Käfiya. Solo en Reino Unido, se conservan 78 manuscritos de obras de Ibn al-Hāăğib y, entre ellos, 24 manuscritos de su al-Käfiya. Cf. Union Catalogue of Manuscripts from the Islamicate World https://www.fihrist.org.uk/catalog/person_29665940. Desconozco el dato global para España, aunque sí conozco la existencia de varios manuscritos de la Käfiya o de comentarios sobre la Käfiya en El Escorial, como, por ejemplo, el ms. n 146, datado de 683/1284 (Derenbourg 1881-1885: I, 8990). Sobre su difusión en el ámbito árabe-islámico véase Ibn Ġazī (2008).

(4) Estas afirmaciones proceden de trabajos recientes de Sartori y Versteegh que deben contrastarse con lo publicado por Binaghi. Esta cuestión queda para otra ocasión, pues no es el objeto de este trabajo.

(5) En adelante BNE.

(6) Ilustración $\mathrm{n}^{\mathrm{o}} 1$.

(7) Ilustración $\mathrm{n}^{\circ} 2$.

(8) Ilustración $\mathrm{n}^{\circ} 3$.

(9) Ilustración $\mathrm{n}^{\circ} 4$.

(10) Nos parece que el manuscrito número 522 de la Biblioteca Universitaria de Salamanca es un ejemplar de la edición de Roma de 1592 de la Käfiya de Ibn al-Hāăğib. Véase Catálogo (1997: 328), donde se describe el contenido del ms. 522, que cuenta con tres obras, la tercera de ellas: " $f$. $111 r-158 v$ : IMPRESO en árabe. IBN AL-HAYIB, Kafiya". 
También resulta de interés que la $\bar{A} \check{g}$ urrümiyya fue conocida entre los moriscos, tal y como lo atestigua la conservación de varias copias de la obra, algunas de ellas con glosas aljamiadas, entre los libros de un morisco aragonés encontrados en Almonacid de la Sierra. Forman parte de esta colección varias traducciones interlineales en aljamiado, como la del documento Esta es el Alcharrumía con su declaración ${ }^{(11)}$. Además, fue traducida al latín. Primero por Petrus Kirstenius (1577-1640), traducción publicada en Roma en 1610, a continuación, por Thomas Erpenius (1584-1624), publicada en Leiden en 1617 y, en tercer lugar, por Tommaso Obicini de Novara (1585-1632), cuya traducción latina de la Ăğurrümiyya fue publicada en Roma en 1631. Peter Kirstenius (1577-1640), doctor en Filosofía, médico del rey de Suecia y profesor de medicina en la Universidad de Uppsala, fue también un políglota autodidacta conocedor del latín, el griego y el siríaco, entre otras lenguas. Estudió también árabe, aplicándose en traducir a Ibn Sīnā, al-Rāzī, Ibn Zuhr, al-Zahrāwī e Ibn Rušd. Su entrega a la impresión de libros en árabe es también bien conocida. Entre 1608 y 1610, publicó su Grammatices arabicae liber I sive orthographia et prosodia arabica (Breslae, 1608); su Liber secundus grammatices arabicae, sive Etymologia arabica (Breslae, 1610); y su Liber tertius grammatices arabicae, sive Syntaxis arabica, (Breslae, 1610), resultando ser su Liber tertius la traducción al latín de la gramática de Ibn Āğurrūm.

En este artículo se presenta un primer acercamiento a este singular volumen preservado en la BNE, que permanece inédito, pero que ha despertado cierto interés en relación con la historia del orientalismo europeo ${ }^{(12)}$. Se concluye que este ejemplar forma parte de una de las etapas del desarrollo de los estudios árabes y orientales en España, que entronca con los inicios del orientalismo europeo, pero que es singular en sí misma debido a la historia de lo que durante siglos fue al-Andalus.

\section{La gramática (al-Kāfiya) de Ibn al-Ḥāğib: contexto y recepción en Europa}

La gramática árabe surge como disciplina autónoma en el siglo II/VIII con influencias de modelos gramaticales griegos recibidos a través de traducciones siriacas. En ella se distinguieron cuatro ramas: al-luga, al-tașrĭf, al-naḥw y al-ištiqāq. Al-luga tiene que ver con el léxico, con su recopilación, su transmisión (naql), su explicación y su registro en obras lexicográficas. Al-tașrîf tiene por objeto la determinación de la forma de la palabra, que procede del registro léxico (luga). Al-naḥw hace referencia a la sintaxis, a la expresión en frases, y concierne el estudio del $i^{i} r \bar{a} b$, es decir la morfemática (harakāt) indicadora de las distintas funciones gramaticales. Al-ištiq $\bar{a} q$ hace referencia a la derivación de las palabras a partir de sus correspondientes raíces.

El ' Ilm al-nahw (Gramática) nace con la primera descripción de la lengua árabe realizada

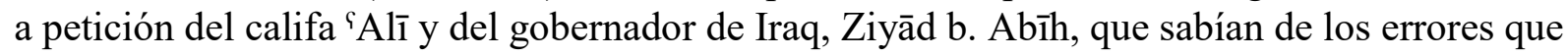
cometían árabes y persas en la recitación del Corán o en su forma de hablar (Pellat 1953: 130). Se acepta que fue Abū 1-Aswad al-Du’alī, fallecido en 69/688 (GAL I, p. 37, SI, p. 72 y Fück 1986), el primero en elaborar una gramática y quien determinó el sistema de signos diacríticos en la escritura del Corán ( $i$ 乌̌ă $m$, marca diacrítica consonántica, y taškīl, marca diacrítica vocálica y otras).

El naḥw se compone de șarf (morfología) e $i^{\zeta} r a \bar{b}$ (sintaxis). Además del 'Ilm al-naḥw (Gramática) otra ciencia, el '́llm al-luga (Lexicografía), surge con el mismo objetivo: la descripción del kalām al-`́arab. El '́llm al-luga incluye la elaboración de vocabularios temáticos

(11) Zanón (1995: 370). J-XII, ff. 160-189.

(12) Rodríguez Mediano (2006: 265, nota 85) ya indicó la existencia de "un borrador de traducción castellana de [...] la Kāfiya de Ibn al-Hāğib, posiblemente del s. XVIr'. El estudio de los manuscritos BNE 7887, 8432 y 8434 de Boyano Guerra y Sánchez-García (2020) augura un conocimiento mucho más preciso del que tenemos en la actualidad sobre el aprendizaje y la enseñanza de la lengua árabe en la España Moderna. 
y de diccionarios, así como la investigación sobre el léxico de la lengua, principalmente las anomalías en la atribución de los alfāz (las palabras) a los $m a^{\varphi} \bar{a} n \bar{\imath}$ (los significados), y la resolución de problemas morfológicos y fonéticos.

Una de las obras de Ibn al-Hạăib es una síntesis de la gramática árabe destinada a la enseñanza de esta lengua en el contexto de la enseñanza formal. El título de la obra es Kāfiya daw̄ al-'arab fì ma'rifa ${ }^{\text {ti }}$ kalām al-'`arab (La [gramática] suficiente para los que necesitan aprender la lengua árabe correcta $\left.{ }^{(13)}\right)$, conocida como al-Kăfiya. Ibn al-Hāğğib estableció la estructura de contenidos de su Käfiya teniendo como modelo el planteado por al-Zamahšarīi ${ }^{(14)}$ en su al-Mufașsal fì 'ilm al-`arabiyyal al-Mufașsal fì al-naḥw (Exposición detallada sobre lingüistica árabe o Exposición detallada sobre gramática) ${ }^{(15)}$, donde el texto se articula atendiendo a las categorías gramaticales. Es decir: nombres (al-asmāi ), verbos $\left(a l-a f^{i} \bar{a} l\right)$ y partículas $(a l-h u r u ̄ f)$. Como Ibn Āğurrūm, Ibn al-Ḥāğib presenta una división de contenidos que hace referencia a tales categorías. Ambas gramáticas se encuadran en el periodo posclásico de la historia de la gramática árabe, un periodo que abarca los siglos VI/XII al IX/XV. Hasta hace poco, el periodo clásico (durante más de un siglo, el único periodo) de la historia de la gramática árabe era el descrito por Flügel (1862), Weil (1913) o Rescher (1925-1933), que reseñaron autores y obras de los siglos I/VII al IV/X, y hasta el siglo V/XII según Sezgin GAS (19672007: IX, 89-94).

Estudio aparte merece la recepción de la gramática árabe en Europa a partir del siglo XVI (Aguiar Aguilar 2019). Uno de los acuerdos del Concilio de Viena (Francia) fue ordenar la fundación de cátedras de hebreo, árabe y caldeo en los estudios o universidades de Salamanca, Oxford, París y Bolonia. Pero, al mismo tiempo, ordenaba prohibir la llamada a la oración y la peregrinación a tumbas de santos musulmanes. Es decir, interesaba conocer la lengua árabe, pero se prohibían manifestaciones del culto islámico. No obstante, el decreto de Vienne relativo a las lenguas semíticas no fue aplicado en la totalidad de los lugares señalados. En Francia, por ejemplo, estas lenguas no se enseñaron hasta el siglo XVI, con la creación por orden de Francisco I del Collège des lecteurs royaux, origen del Collège de France (Chiron 2011 y Girard 2013).

Según Troupeau (1962: 359)(16), el corpus de gramáticas de la lengua árabe que conocieron, estudiaron y tradujeron los orientalistas del siglo XVI -momento en que se considera que nacen los estudios árabes en el resto de Europa- estaba compuesto por cuatro obras: la Āğurrümiyya, la Kāfiya de Ibn al-Hāāğib, al-Taṣrīf de 'Izz al-Dīn al-Zanğānī, también conocido como al-Tașrīf

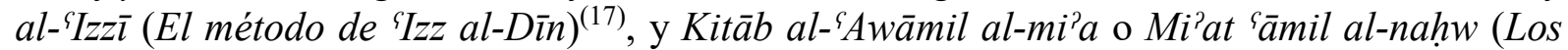
cien [elementos] de rección gramatical) de al-Ğurğānī ${ }^{(18)}$. Sin embargo, si tomamos como

(13) Es decir, "la lengua árabe libre de corrupción que pretendian describir los sabios musulmanes" (Peña 19891990: 215).

(14) Nacido en 467-8/1075 y fallecido en 538/1144. Agius 1982 y Versteegh 2002.

(15) Materia de reflexión para otro momento es la recepción mālikí de la gramática de al-Zamahšarī, teólogo mu'tazilí en desacuerdo con situar el persa como lengua vehicular en lugar del árabe, en una perspectiva no šu'ūbí.

(16) Aunque el título del artículo de Troupeau hace pensar en un estudio de las traducciones latinas de $a l$ Muqaddima al-āğurrūmiyya, en realidad se trata de un breve listado de algunos tecnicismos gramaticales y su correspondiente traducción al latín.

(17) Activo ca. 625/1228. Su gramática fue también editada y traducida al latín por Giovanni Battista Raimondi, publicándose en Roma en 1610 con el título Liber Tasriphi compositio est senis Alemami [= Kitāb al-tașrīf ta'lĭf al-šayj al-imām]. Traditur in eo compendiosa notitia coniugationum uerbi Arabici. Sobre al-Zanğān̄̄, véase $E I^{2}$ s.v. y Schnurrer (1811: $\left.\mathrm{n}^{\circ} 47\right)$.

(18) Fallecido en 471/1078. Versión latina: Libellus centum regentium, edición de Thomas Erpenius, Leiden, 1617, publicado en el mismo volumen junto con la Ā̆grrūmiyya. 
referencia la biblioteca del humanista flamenco del siglo XVI Niklaas Cleynærts Beka(19) (Bakelants y Hoven 1981; Torre Giménez 1998: 76 y Tulkens 2015), estudiante de hebreo y griego en Lovaina y llegado a España en 1530-1531 con el encargo de organizar la Biblioteca Colombina de Sevilla, las gramáticas de la lengua árabe que habría adquirido en Fez ca. 1540 serían las primeras que circularon por Europa (Loop 2017: 3). Son las siguientes:

- El Kitāb al-ğumal fi l-naḥw (El libro de lo esencial sobre gramática o Compendio de gramática) de Abū 1-Qāsim al-Zağğāăği ${ }^{(20)}$.

- El Šarḥ al-Mufașṣal, comentario del al-Mufașsal fi silm al-`arabiyya/ al-Mufașal fi al-naḥw (Exposición detallada sobre gramática o Exposición detallada sobre lingüística árabe) de al-Zamahšarī ${ }^{-21)}$.

- La Ăğurrūmiyya.

Se venía considerando que había sido el Mufașșal de al-Zamahššrī la gramática árabe que había llegado a la academia europea en el siglo XIX, al ser el texto base utilizado por Caspari para elaborar su gramática de la lengua árabe publicada en 1848 y que sería a su vez la base de las gramáticas de la lengua árabe occidentales a partir de la traducción al inglés realizada por Wright (Versteegh 2002). Pero estudios más recientes han hecho posible encontrar y comprender otras vías de introducción de la gramática árabe en Occidente, como por ejemplo el interés por la lengua árabe en España en el siglo XVI y XVII.

La primera edición publicada en Europa de la Kăfiya de Ibn al-Hāğib fue la Grammatica arabica, dicta Caphia, auctore filio Alhagiabi, impresa por la Typographia Medicea en Roma en $1592^{(22)}$, publicada junto con la Grammatica arabica in compendium redacta, qua vocatur Giarrumia, auctore Mahmeto filio Dauidis Alsanhagij, es decir, la gramática árabe de Ibn Āğurrūm o Āğurrūmiyya ${ }^{(23)}$ (Aguiar Aguilar 2019). Se trata uno de los textos árabes publicados por la Typographia Medicea Orientale bajo la dirección de Giovanni Battista Raimondi ${ }^{(24)}$ entre los que se encontraba también el Alphabetum Arabicum $^{(25)}$ (Jones 1994: 88-89).

\section{El ejemplar de la Kāfiya de Ibn al-Ḥāğib (Roma, 1592) de la BNE}

El ejemplar de la edición de Roma de 1592 de la Āğurrūmiyya y la Kāfiya de Ibn al-Hāăğib que forma parte de los fondos de la $\mathrm{BNE}^{(26)}$ se conserva en buen estado. Su versión digital está disponible en línea. Es un ejemplar de la editio princeps de ambas obras gramaticales ${ }^{(27)}$.

(19) Latinizado Nicolaus Clenardus (también Nicolas Clénard, Nicolau Clenardo, Nicolas Clenardus, Nicolaus Clenardus, Nicolaus Cleonardus, Nicolas Clenardus). En 1512, ingresó en la Universidad de Lovaina con el nombre de Nicolaus de Beka de Diest. Nacido en 1495 en Diest (Vlaams-Brabant, Bélgica) y fallecido en 1542 en Granada y enterrado, quizás, en la Alhambra.

(20) Fallecido ca. 337/948-9. Gramático $m u^{\varsigma}$ tazilī del que conocen solo algunas anécdotas de su vida.

(21) Nacido en 467/1075 en Zamaȟšar (Hwārizm) y fallecido en 538/114 en Ğurğāniyya. Intelectual hanafí $m u^{`}$ tazilī autor de obras de exégesis, tradición, teología, jurisprudencia, gramática, lexicografía y literatura.

(22) In 4, consta de 96 páginas. Schnurrer (1811: $\left.\mathrm{n}^{\circ} 42\right)$.

(23) In 4 , consta de 12 páginas. Schnurrer $\left(1811, \mathrm{n}^{\circ} 43\right)$.

(24) Nacido en 1536 y fallecido en 1614.

(25) Schnurrer $\left(1811, \mathrm{n}^{\mathrm{o}} 41\right)$.

(26) El documento fuente del presente artículo es el manuscrito 7887 de la BNE. Su reproducción digital está disponible en la Biblioteca Digital Hispánica en línea en el siguiente enlace: http://bdhrd.bne.es/viewer.vm? $\mathrm{id}=0000173523 \&$ page $=1$. Véase un comentario codicológico y de contenido del ms. BNE 7887 comparado con los mss. BNE 8432 y 8434 en Boyano Guerra y Sánchez-García (2020).

(27) A partir de la impresión de Roma de 1592, la Kāfiya de Ibn al-Ḥāğib fue publicada en múltiples ocasiones, especialmente en Estambul. Véase GAL I, 367. 
La tipografía utilizada es la desarrollada por Robert Granjon ${ }^{(28)}$ entre 1580 y 1586 . Está impreso de tal forma que da la sensación de que es un manuscrito a doble tinta (roja para títulos y negra para texto), con 13 líneas por página, reclamo en cada página y número de pliego (se imprimió in-octavo), producto de una perfecta técnica que supera y mucho a los primeros libros impresos con caracteres móviles árabes (Aguiar Aguilar 2019) ${ }^{(29)}$.

Como señala Smitskamp (1976: vol. I, $\mathrm{n}^{\mathbf{0}}$ 30), algunos ejemplares de la edición de Roma de 1592 de la Ăğurrūmiyya y la Kăfiya de Ibn al-Hâăğib presentan el título en latín, el lugar de impresión, el nombre de la imprenta y el año en la página de título de cada gramática. No es el caso del ejemplar que nos ocupa ${ }^{(30)}$.

El libro perteneció a Pedro de Castro Vaca y Quiñones (m. 1623), arzobispo de Granada (1590-1610) y Sevilla (1610-1623) (Boyano Guerra y Sánchez-García 2020) y formaba parte de los más de 200 ejemplares de la biblioteca del conde de Miranda, adquiridos en 1757 por 5.500 reales de vellón (Andrés 1979: 612-613) ${ }^{(31)}$ para integrarse en los fondos de la Real Biblioteca fundada en 1711 por orden del rey Felipe V, origen de la BNE. Es posible que el ejemplar entrara en la biblioteca familiar en vida de Juan López de Zúñiga (n. 1551-m. 1608), conde de Miranda, capitán general de Cataluña, presidente del Consejo Real de Castilla y virrey de Nápoles (entre 1585 y 1596). Felipe III le nombró Consejero de Estado el 16 de septiembre de 1598, pocos días después del comienzo de su reinado, pidiendo el retiro de la corte en 1608, año de su fallecimiento (Andrés 1979: 614; Vargas Hidalgo 1995: 433-435; y Gómez Rivero 2003: 195, nota 9).

En el Catálogo de los manuscritos árabes de la Biblioteca Nacional de Madrid de F. Guillén Robles (1889: 245), el documento está identificado con el número DXCIX y se describe como sigue ${ }^{(32)}$ :

\section{DXCIX}

ACHARRUM (IBN), Moh. ben Daud Az-Zinhachí.

كتاب الاجرومية في النحو

Libro (titulado) la Acharrumia, que trata de la gramática.

Impreso y ms.: $4 .^{\circ}$

Comienza el texto: .... قال الثيخ الامام العالم

Concluye el vol.: فيرد ما حدف المفتوح ما قبلها تقلب الفام المات

135 fol. entre bl. y útiles, impresos y mss.: algunos foliados: en castellano y árabe: éste en carácter oriental: el castellano, ya escrito entre líneas, ya aparte en folios, en $4 .^{\circ}$, y $4 .^{\circ}$ marquilla apaisado, letra de fines del s. XVII: anot. marg.: 3 fol. bl. al fin: encuad. en pergamino.

Comprende, en 41 fol., una edición en ár., impresa, de la obra del mencionado autor, repetidamente citado en este Catál., traducida en parte entre líneas, en latín y castellano, y por separado al principio, en castellano.

(28) Nacido en París en 1513 y fallecido en Roma en 1589

(29) El primer libro impreso con caracteres árabes fue el libro de horas del rito bizantino melquita Kitāb șalāt alsawā $\varsigma_{\bar{l}}$ bi-hasāb țaqs al-kanīsat al-Iskandariyya (Libro de horas según el cómputo de la liturgia de la Iglesia de Alejandría), impreso por Gregorio de Gregorio en Fano (Italia) en 1514. El primer libro sobre tipos de escrituras de oriente se debe a Guillaume Postel (Linguarum duodecim characteribus differentium alphabeta. Introductio ac legendi modus longe facillimus, Paris, 1538). La tipografía es bastante deficiente en ambos impresos. En la Biblioteca Histórica "Marqués de Valdecilla" de la Universidad Complutense de Madrid se conserva uno de los 15 ejemplares que se conservan en el mundo. Véase Torres Santo Domingo (2014).

(30) Ilustración $\mathrm{n}^{\mathrm{o}} 1$.

(31) El ejemplar fue vendido siendo don Antonio López de Zúñiga el XIII conde de Miranda, que no parece haber sido un bibliófilo y que recibió la biblioteca familiar de su padre, don Joaquín López de Zúñiga, XII conde de Miranda. El artículo de Gregorio de Andrés explica en detalle los pormenores de la distribución testamentaria de la biblioteca familiar.

(32) Transcribo literalmente. 
Contiene además:

2. ALHACHIB (IBN).

Comprende el resto del ms. la Cafia del mencionado autor, también muy citada en este Catál., con una traducción literal castellana, escrita en letra del s. XVII, interpolada folio á folio en el volumen.

Procedente de la librería del Conde de Miranda.

Gregorio de Andrés (1979: 624, $\mathrm{n}^{\mathrm{o}} 88$ ) hace mención al manuscrito árabe $\mathrm{n}^{\mathrm{o}} 7887$ de la BNE como "Alfurrumia, (Libro de la Ayarumía sobre la gramática), en arábigo traducido en romance en 15 r. $=7887$ (impreso)" y también como "Un manuscrito arábigo en 15 r. $=7887$ " (1979: 626, $\mathrm{n}^{\mathrm{o}}$ 186), perteneciente a la biblioteca del conde de Miranda, lo que no deja de resultar incongruente, pues en el primer caso lo cita como libro en cuarto y en el segundo caso como libro en octavo, de ahí que encontremos el "manuscrito" 7887 citado dos veces en la catalogación de Gregorio de Andrés.

En el Inventario BNE (1988: XII, 194), el manuscrito 7887 se describe del siguiente modo:

\section{7}

Muhamad b. Dawd Assinhaği, AĞARŪM: Libro de la Ağarūmīa sobre la gramática.- S. XVII (?), papel, 220/310 x 150/210 mm., $136 \mathrm{ff}$.

Edición árabe impresa y su traducción interlineal, parcial, en latín y castellano del siglo XVII. Además contiene la Kāfia de ibn Alhāğib con traducción literal castellana, folio a folio.

Pos.: Conde de Miranda.

F. GUILLEN ROBLES, Catálogo de los mss. árabes de la B. N. de Madrid, p. 245, n. DXCIX. ANDRES, Miranda, p. 621.

López-Baralt e Iversen (2006) se ocuparon de la contextualización de los manuscritos BNE 7887, 8432 y 8434, pero recientemente Boyano Guerra y Sánchez-García (2020) han situado estos ejemplares en el entorno granadino de la traducción de los libros plúmbeos de finales del siglo XVI y principios del XVII, refutando la tesis de López-Baralt e Iversen y confirmando la de García-Arenal y Rodríguez Mediano (2017).

\section{Conclusiones}

El libro de la biblioteca del conde de Miranda incorporado en 1757 a los fondos de la BNE e identificado con el número 7887 de su colección de manuscritos árabes es un volumen mixto que contiene texto impreso y texto manuscrito. Se trata de un ejemplar de la edición de Roma de 1592 de las gramáticas de Ibn Āğurrūm y de Ibn al-Ḥāğib al que le falta la portada que sí está en los ejemplares conservados en la Biblioteca Nazionale di Napoli Vittorio Emanuele III y en la Österreichische Nationalbibliothek. El volumen contiene hojas de papel sueltas, anotaciones al margen y escritura interlineal. Estas hojas, notas marginales y anotaciones interlineales (en español y algunas palabras en griego y en hebreo) presentan diversos estados de traducción de ambas gramáticas de varias manos. Sorprende la complejidad de este volumen, que abre tantas perspectivas de estudio sobre aspectos lingüísticos y filológicos. Pero, además, sobre su significado en el contexto del orientalismo español del siglo XVII (Rodríguez Mediano 2006), pues el denominado por Gregorio de Andrés “Alfurrumia, (Libro de la Ayarumía sobre la gramática), en arábigo traducido en romance en 15 r. = 7887 (impreso)" (Andrés 1979: $624, n^{\circ} 88$ ) no es el único ejemplar de la biblioteca del señor conde de Miranda a través del cual observamos el interés por la lengua árabe en España en el siglo XVII. Sin duda este ejemplar forma parte de una de las etapas del desarrollo de los estudios árabes y orientales en España, que entronca con los inicios del orientalismo europeo, pero que es singular en sí misma debido a la historia de lo que durante siglos fue al-Andalus. Inmerso como estuvo don Juan de Zúñiga en el episodio propagandístico de los libros plúmbeos del Sacro Monte y en la discusión sobre 
la definición dogmática de la Concepción, sus libros revelan dimensiones más amplias que una mera presencia de unas gramáticas de la lengua árabe en su biblioteca. Formaban parte de ella también diversos cuadernos con anotaciones de distintas temáticas: un "Vocabulario arábigo en $20 \mathrm{r} .=7888$ " (Andrés 1979: 624, $\mathrm{n}^{\circ}$ 72), un "Alphabeto arábigo en $15 \mathrm{r} .=8434$ " (Andrés 1979: 624, $\mathrm{n}^{\circ}$ 80), "Varios autores árabes en cuarto en $15 \mathrm{r} .=7453$ " (Andrés 1979: 626, $\mathrm{n}^{\circ}$ 180), "Evangelios y Epistolas en árabe = 4971" (Andrés 1979: 627, n 31), "Una traducción latina y castellana de los libros de plomo hallados en el Sacromonte de Granada $=6637$ " (Andrés 1979: 627, $\mathrm{n}^{\mathrm{o}}$ 50) ...

Pro captu lectoris habent sua fata libelli.

\section{Referencias bibliográficas}

AgIUs, Dionisius A. (1982): "Some Bio-bibliographical Notes on Abū-l-Qāsim Maḥmūd b. ‘Umar al-Zamakhshārī”, Al-`Arabiyya (Journal of the American Association of Teachers of Arabic), $\mathrm{n}^{\mathrm{o}} 15 / 1-2$, pp. 108-130.

AguiAr Aguilar, Maravillas (2019): "Nam loquens lingua...: La tradición latina de la gramática de Ibn Âŷrrūm (m. 723/1323) de los siglos XVI y XVII", Fortunatae, no 29 , pp. 7-26 http://doi.org/10.25145/j.fortunat.2019.29.001 .

ANDRÉS, Gregorio de (1979): "Los códices del conde de Miranda en la Biblioteca Nacional", Revista de Archivos, Bibliotecas y Museos, nº 82, pp. 611-628.

BaKelants, Louis y Hoven, René (1981): Bibliographie des oeuvres de Nicolas Clénard, 1525-1700, Verviers: P.-M. Gason, 2 vols.

Ben CHENEB, Mohammed (1913-1936): “Ibn Ādjurrūm”, s.v. en Encyclopaedia of Islam. First Edition, Leiden: Brill.

Boyano Guerra, Isabel y SÁnchez-GarcíA, Patricia (2020): "Una biblioteca en los márgenes: Pedro de Castro aprende árabe”, Al-Qanțara, $\mathrm{n}^{\circ}$ XLI-2, pp. 517-544. https://doi.org/10.3989/alqantara.2020.014.

BrockelmanN, Carl, Geschichte der arabischen Litteratur (1898, 1902). Edición original: Volumen I, Weimar: E. Felber, 1898. Volumen II, Berlin: E. Felber, 1902. Suplementos = GAL S (1937-1942): Supplementbände, Leiden: Brill, 3 vols. Segunda edición corregida (1909): Leipzig: C. F. Amelang. Tercera edición con suplementos (1943-1949): Leiden: Brill, 5 vols. Reimpresión (1996): Leiden: Brill, 5 vols. y (2012): 6 vols. Traducción al inglés de J. Lameer: Leiden-Boston: Brill, 2016 (vol. I), 2017 (vol. II), 2017 (suplemento I) y 2018 (suplemento II). (= GAL)

CASPARI, Carl Paul (1848): Grammatica arabica in usum scholarum academicarum. Accedit brevis chrestomathia ex codd. mscr. Concinnata, Lipsiae: Sumtibus C. L. Fritzschii.

Catálogo (1997): Catálogo de manuscritos de la Biblioteca Universitaria de Salamanca. I Manuscritos 1-1679bis, Salamanca: Ediciones Universidad de Salamanca.

Chiron, Yves (2011): Histoire des conciles, Paris: Perrin.

DERENBOURG, Hartwig (1881-1885): Les manuscrits arabes de l'Escurial. Paris: Imprimerie Nationale. Reimpresión (1970): Hildesheim-New York: Georg Olms Verlag.

$E I^{2}=$ Encyclopédie de l'Islam, $2^{\mathrm{a}}$ ed., 12 volúmenes. Leiden: Brill, 1960-2006.

FLEISCH, Henri (1986): "Ibn al-Ḥādjib”. En $E I^{2}$, vol. III, p. 781.

FLÜGEL, Gustav (1862): Die grammatischen Schulen der Araber. Leipzig: F. A. Brockhaus. Reimpresión: Nendeln, Kraus Reprint, 1966.

FÜCK, Johann Wilhelm (1986): “Abū l-Aswad al-Du’alî”. En $E I^{2}$, vol. I, pp. 106-107. Véase Monique Bernards, s.v. en K. Fleet et al., Encyclopaedia of Islam, THREE, Leiden: Brill, en línea. 
García-Arenal, Mercedes y Rodríguez Mediano, Fernando (2017): "Sacred History, Sacred Languages: The Question of Arabic in Early Modern Spain”. En Jan Loop, Alastair Hamilton y Charles Burnett (eds.). The Teaching and Learning of Arabic in Early Modern Europe. Leiden-Boston: Brill, pp. 133-162.

GAS = Sezgin, Fuat (1967-2007): Geschichte des arabischen Schrifttums. Leiden: Brill.

GÓMEz Rivero, Ricardo (2003): "Lerma y el control de cargos", Anuario de Historia del Derecho Español, n 73 , pp. 193-230.

Guillén RoBles, Francisco (1889): Catálogo de los manuscritos árabes existentes en la Biblioteca Nacional de Madrid, Madrid: Manuel Tello.

GIRARD, Aurélien, (2013): "Les manuels de langue arabe en usage en France à la fin de l'Ancien Régime". En S. Larzul y A. Messaoudi (dir.), Manuels d'arabe d'hier et d'aujourd'hui. France et Maghreb, XIXe-XXIe siècle, Paris : Bibliothèque Nationale de France, pp. 12-26.

IBN ĠAZI, Āmāl Hुalīfa (2008) : Ğuhūd al-raḍ̂̄ l-lugiawiyya fì šarhayhi``alā Muqaddimatay Ibn al-Hāăgib, Sirt, Lībiyā: Mağlis al-țaqāfa al-'`āmm.

Inventario BNE (1988): Inventario general de manuscritos de la Biblioteca Nacional, Madrid.

JONES, Robert (1994): "The Medici oriental press (Rome 1584-1614) and the impact of its Arabic publications on northern Europe". En G. A. Russell (ed.). The 'Arabick' interest of the Natural Philosophers in seventeenth-century England, Leiden: Brill, pp. 88-108.

Loop, Jan et al. (eds.) (2017): The Teaching and Learning of Arabic in Early Modern Europe, Leiden-Boston, Brill.

LÓPEZ-BARALt, Luce e IVERSEN, Reem (colab.) (2006): "A zaga de tu huella". La enseñanza de las lenguas semíticas en Salamanca en tiempos de san Juan de la Cruz, Madrid, Trotta.

Pellat, Charles (1953): Le milieu bașrien et la formation de Ğăhiz, Paris: AdrienMaisonneuve.

PEÑA, Salvador (1989-1990): "Sobre la llamada lexicografía árabe: 'Ilm al-luga", Miscelánea de Estudios Árabes y Hebraicos, nº 38-39 (volumen doble), pp. 213-233.

RESCHER, Oskar (1925-1933): Abriss der arabischen Litteraturgeschichte, Stuttgart, s.n. Reimpresión: Osnabrück, Biblio Verlag, 1983.

Rodríguez Mediano, Fernando (2006): "Fragmentos de orientalismo español del s. XVII", Hispania. Revista Española de Historia, vol. LXVI, n 222, enero-abril, pp. 243-276.

SARTORI, Manuel (2013): Le Šarh al-Kāfiyat de Ibn al-Hāğgib: Edition critique d'un manuscrit grammatical arabe du VIIe/XIIIe siècle. Thèse de doctorat. Université de Provence.

SCHNURRER, Christian Friederich von (1811): Bibliotheca arabica, Halae ad Salam: Typis et sumtu I. C. Hendelii. Reimpresión: Amsterdam, Oriental Press, 1968.

SMitsKAMP, Rijk (1976-1991): Philologia orientalis: A description of books illustrating the study and printing of oriental languages in $16^{\text {th }}$ and $17^{\text {th }}$-Century Europe, Leiden: Brill, 3 vols.

TORRE GIMÉNEZ, Estrella de la (1998): "Nicolas Clénard, un arabista flamenco del siglo XVI". En Estudios de la Universidad de Cádiz ofrecidos a la memoria del profesor Braulio Justel Calabozo, Cádiz: Universidad de Cádiz, pp. 71-76.

TORRES SANTO DOMINGO, Nuria (2014): "500 años del primer libro impreso en caracteres móviles árabes", Pecia Complutense, no 21, pp. 1-20.

TroupEAU, Gérard (1986): "Ibn Ādjurrūm", s.v. en $E I^{2}$.

Troupeau, Gérard (1962): “Trois traductions latines de la Muqaddima d'Ibn Āğurrūm”. En Études d'orientalisme dédiées à la mémoire de Lévi-Provençal, Paris: Maisonneuve et Larose, tomo I, pp. 359-365.

TulKens, Joris (2015): "Nicolaes Cleynaerts". En D. Thomas (ed.). Christian-Muslim Relations 1500 - 1900, Leiden, vol. 6, pp. 125-136. 
Versteegh, Cornelis Henricus Maria “Kees” (2002): “al-Zamakhsharī”. En $E I^{2}$, vol. XI, pp. 432-434.

WeIL, Gustav (1913): Die grammatischen Streifragen der Basrer und Kufer, Leiden: Brill.

ZANÓN, Jesús (1995): "Los estudios de lengua árabe entre los moriscos aragoneses a través de los Manuscritos de la Junta", Sharq al-Andalus, n 12, pp. 363-374. 


\section{ILUSTRACIONES}

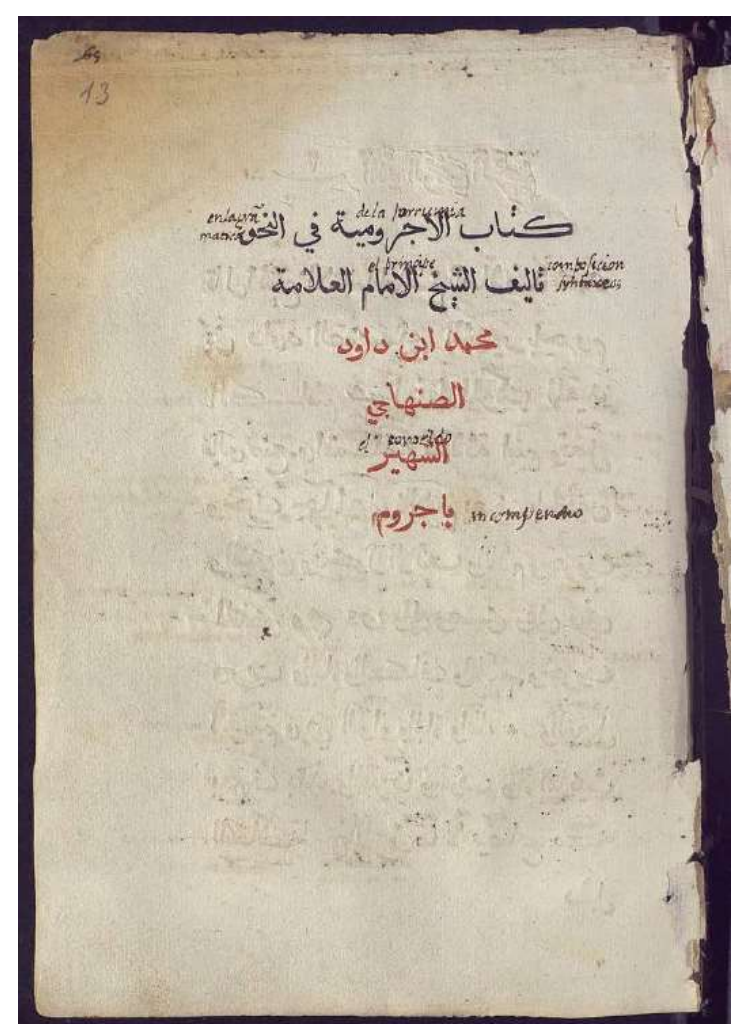

Portada de la edición de Roma de 1592 de la Āğurrūmiyya. Ejemplar de la Biblioteca Nacional de España. Fuente: BNE http://bdhrd.bne.es/viewer.vm?id=0000173523\&page=1. Licencia CC-BY-NC-SA.

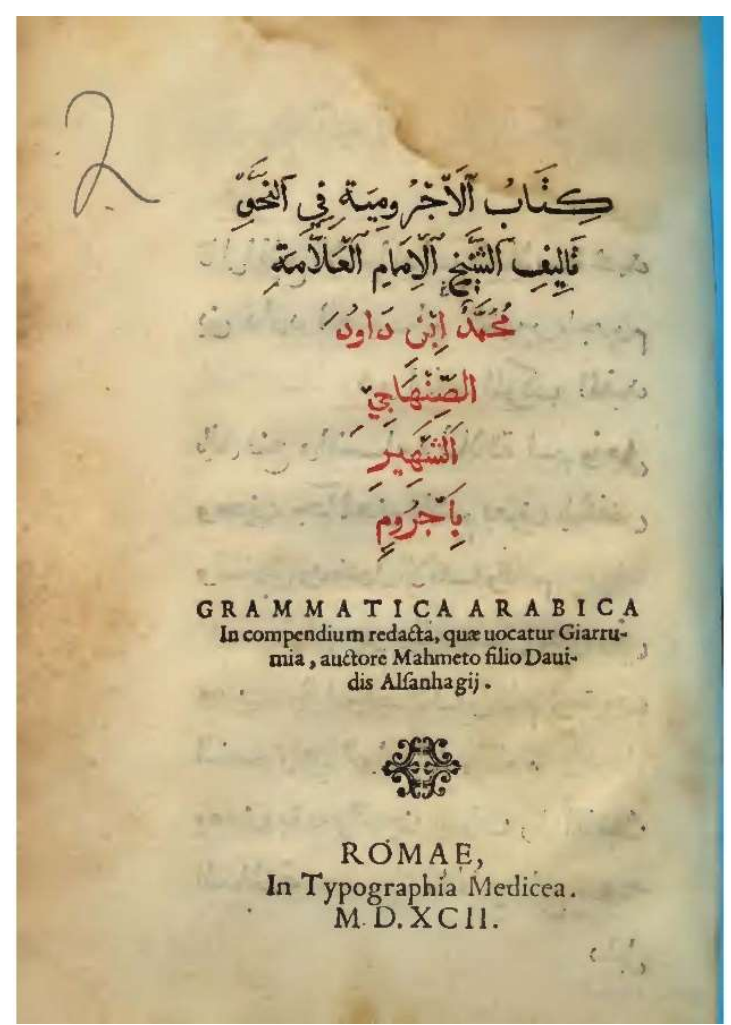

Portada de la edición de Roma de 1592 de la Āğurrūmiyya. Ejemplar de la Biblioteca Nazionale Vittorio Emanuele III (Nápoles). Fuente: www.archive.org (Public Domain Mark 1.0 Creative Commons).

ILUSTRACIÓN N ${ }^{\circ} 1$

Portadas de los ejemplares de la edición de Roma de 1592 de la Āğurrūmiyya conservados en Madrid y Nápoles. 


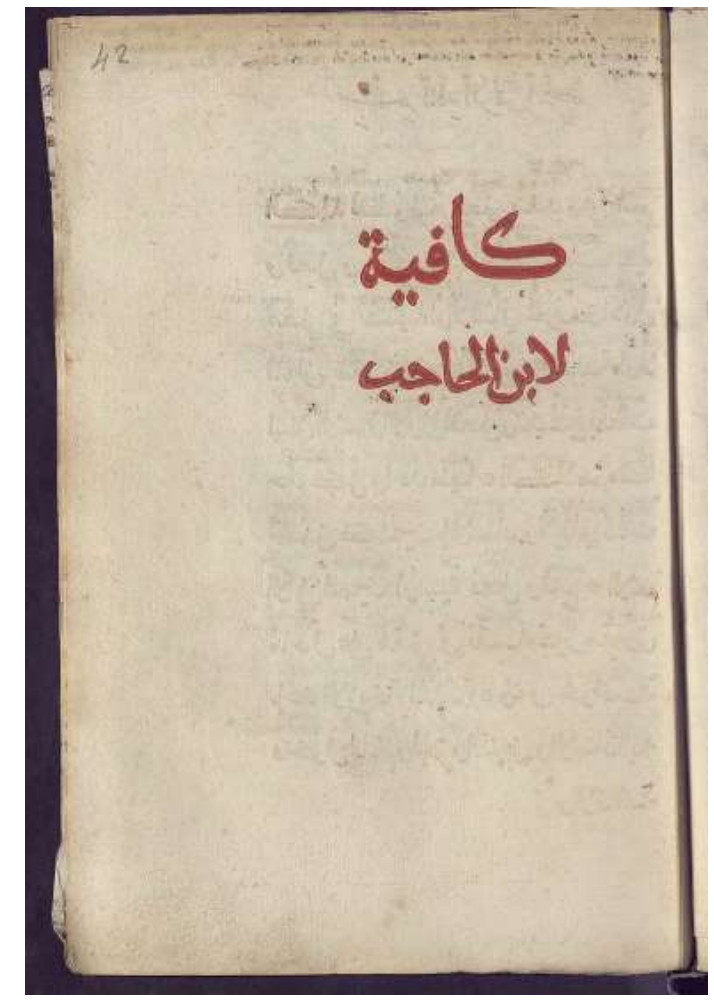

Portada de la edición de Roma de 1592 de la Käfiya. Ejemplar de la Biblioteca Nacional de España, procedente de la biblioteca del conde de Miranda. Fuente: BNE http://bdh-

$\underline{\text { rd.bne.es/viewer.vm? } \mathrm{id}=0000173523 \& \text { page }=1}$. Licencia CC-BY-NC-SA.

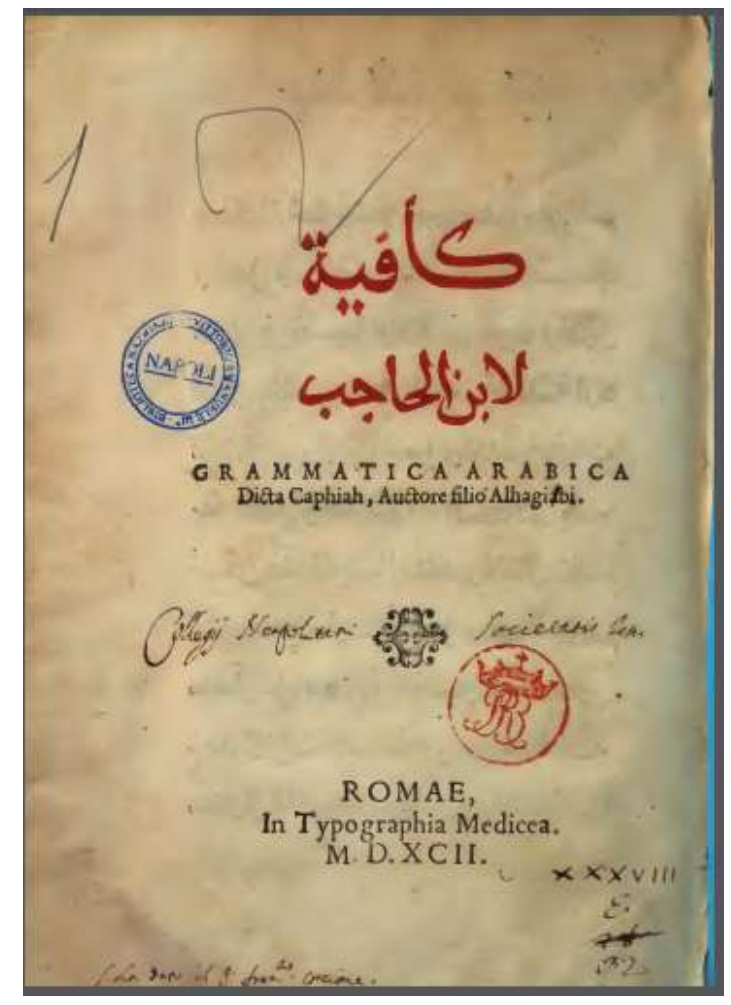

Portada de la edición de Roma de 1592 de la Kāfiya. Ejemplar de la Biblioteca Nazionale Vittorio Emanuele III (Nápoles). Fuente: www.archive.org (Public Domain Mark 1.0 Creative Commons).

\section{ILUSTRACIÓN No2}

Portadas de los ejemplares de la edición de Roma de 1592 de la Kāfiya conservados en Madrid y Nápoles. 


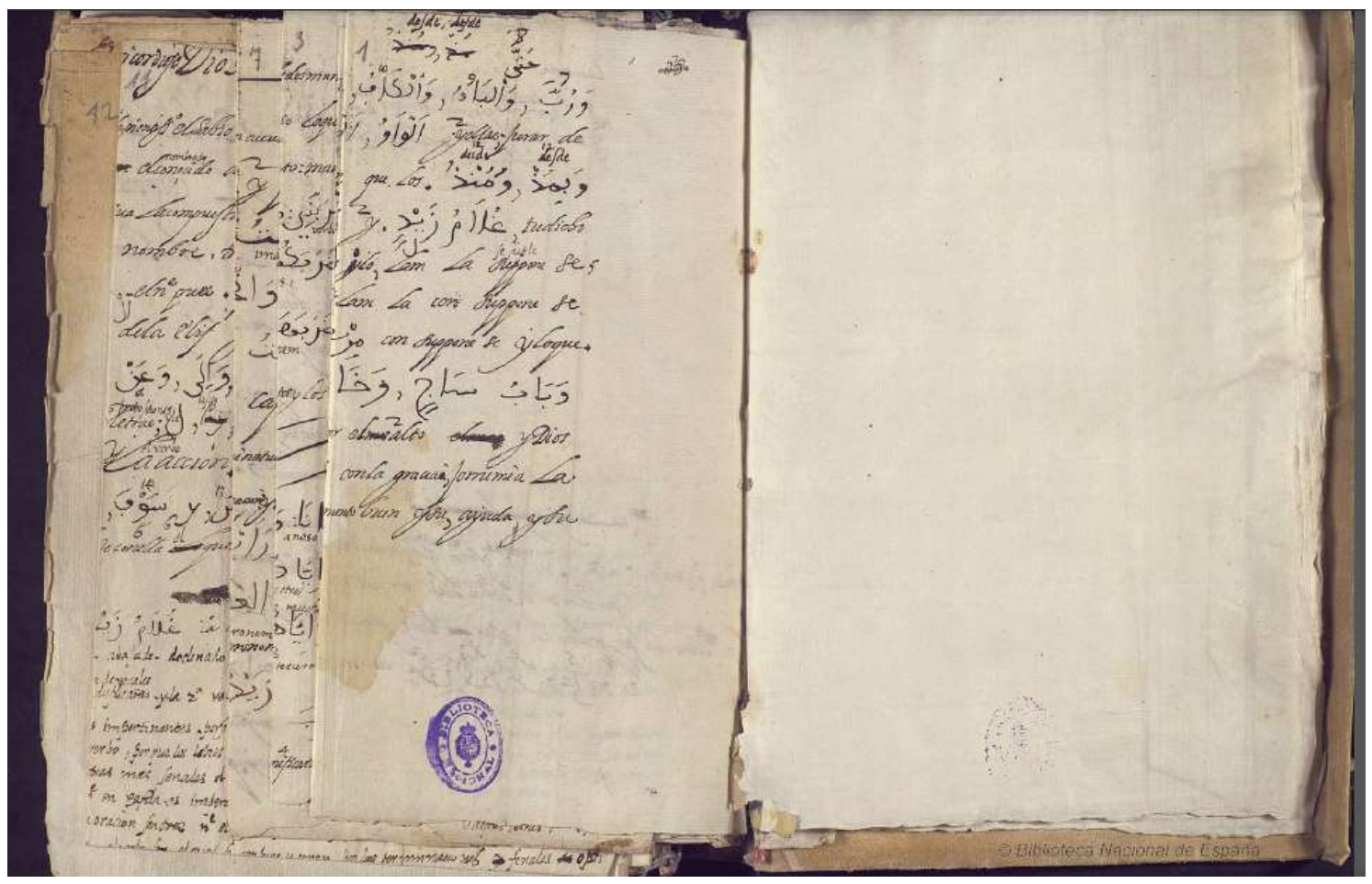

ILUSTRACIÓN No 3

Inicio del manuscrito árabe $n^{\circ} 7887$ de la Biblioteca Nacional de España tal y como se presenta en la copia digitalizada que proporciona la institución y donde se observa la existencia de un número indeterminado de hojas de papel plegadas. Fuente: BNE http://bdh-rd.bne.es/viewer.vm?id=0000173523\&page=1. Licencia CC-BY-NCSA. 

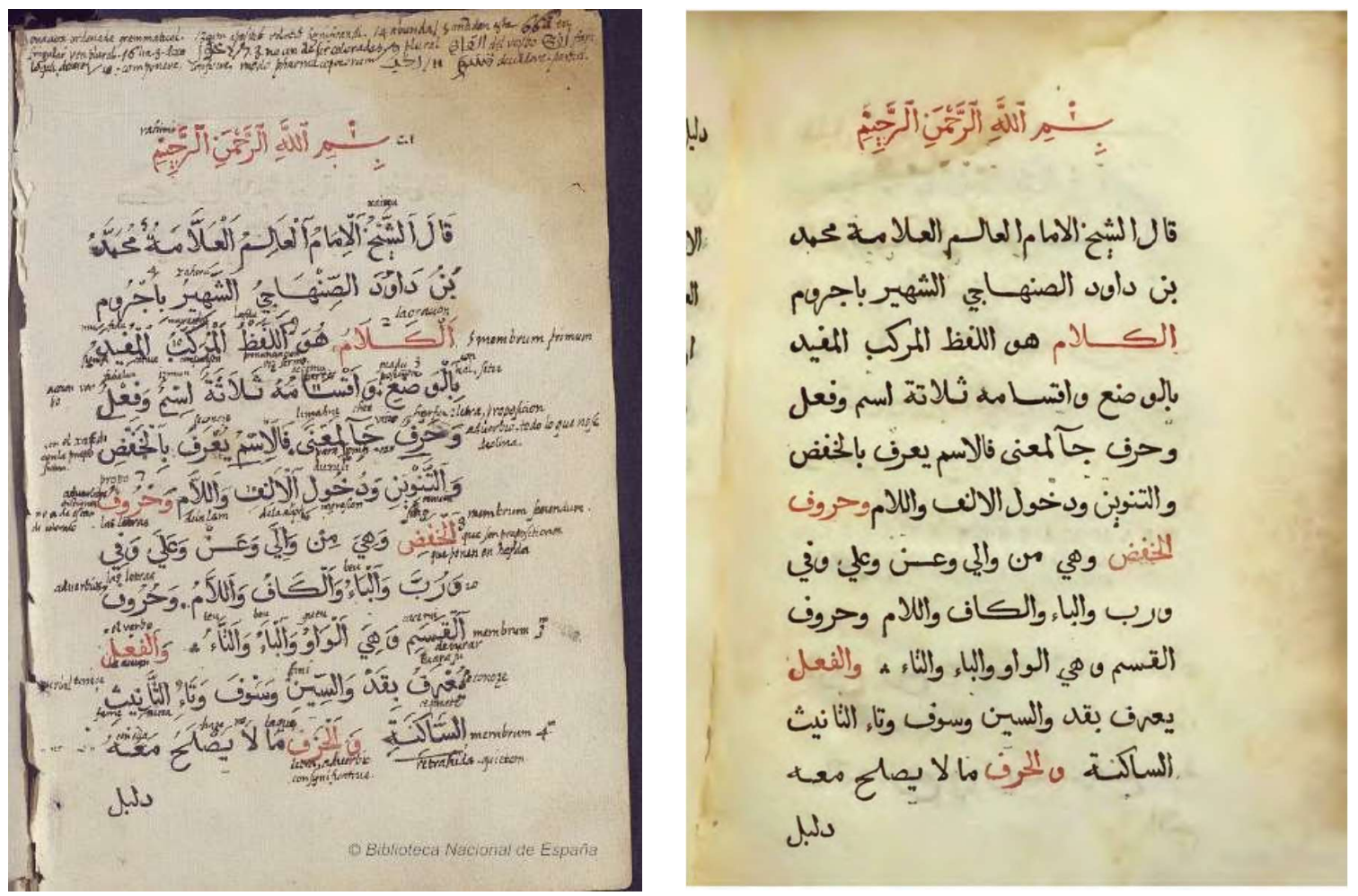

\section{ILUSTRACIÓN No 4}

Izquierda: Primera página de la edición de Roma de 1592 de la Ăğurrümiyya, perteneciente a la biblioteca del conde de Miranda, catalogada por Guillén Robles como manuscrito árabe 7887 de la Biblioteca Nacional de España. Presenta traducción interlineal al español, nota en margen superior escrita en español y varias anotaciones en margen derecho en latín (membrum primum, membrum secundum, membrum 3 [tertiu]m y membrum 4 [quartu] $m$ ) que corresponden no a una traducción latina, sino al uso de ciertos tecnicismos gramaticales. Fuente: BNE http://bdh-rd.bne.es/viewer.vm?id=0000173523\&page=1. Licencia CC-BY-NC-SA.

Derecha: Primera página de la edición de Roma de 1592 de la Ăğurrūmiyya. Ejemplar de la Biblioteca Nazionale Vittorio Emanuele III (Nápoles), sin anotaciones. Fuente: www.archive.org (Public Domain Mark 1.0 Creative Commons). 